SUBJECT AREAS:

ENZYME MECHANISMS

BIOLOGICAL PHYSICS

Received

29 July 2014

Accepted

21 November 2014

Published

12 December 2014

Correspondence and requests for materials should be addressed to Z.W. (wuzy@ustc.edu. cn);W.C. (chuws@ ustc.edu.cn) or W.G. (wgong@sun5.ibp.ac.

$\mathrm{cn})$

\footnotetext{
* These authors contributed equally to this work.
}

\title{
How water molecules affect the catalytic activity of hydrolases - A XANES study of the local structures of peptide deformylase
}

\author{
Peixin Cui 1,3*, Yu Wang ${ }^{2 *}$, Wangsheng Chu ${ }^{1,3}$, Xiaoyun Guo ${ }^{3,4}$, Feifei Yang ${ }^{1}$, Meijuan Yu ${ }^{3}$, Haifeng Zhao ${ }^{3}$, \\ Yuhui Dong ${ }^{3}$, Yaning Xie ${ }^{3}$, Weimin Gong ${ }^{4,5} \&$ Ziyu Wu ${ }^{1,3}$

\begin{abstract}
${ }^{1}$ National Synchrotron Radiation Laboratory, University of Science and Technology of China, Hefei, China, ${ }^{2}$ Shanghai Synchrotron Radiation Facilities, Shanghai Institute of Applied Physics, Chinese Academy of Sciences, Shanghai, China, ${ }^{3}$ Institute of High Energy

${ }^{5}$ Hefei National Laboratory for Physical Sciences at the microscale, University of Science and Technology of China.
\end{abstract} \\ Physics, Chinese Academy of Sciences, Beijing, China, ${ }^{4}$ Institute of Biophysics, Chinese Academy of Sciences, Beijing, China,
}

Peptide deformylase (PDF) is a prokaryotic enzyme that catalyzes the deformylation of nascent peptides generated during protein synthesis and water molecules play a key role in these hydrolases. Using X-ray absorption near edge spectroscopy (XANES) and ab initio calculations we accurately probe the local atomic environment of the metal ion binding in the active site of PDF at different $\mathrm{pH}$ values and with different metal ions. This new approach is an effective way to monitor existing correlations among functions and structural changes. We show for the first time that the enzymatic activity depends on $\mathrm{pH}$ values and metal ions via the bond length of the nearest coordinating water (Wat1) to the metal ion. Combining experimental and theoretical data we may claim that PDF exhibits an enhanced enzymatic activity only when the distance of the Wat1 molecule with the metal ion falls in the limited range from 2.15 to $2.55 \AA$.

P eptide deformylase (PDF, EC 3.5.1.27) is an important member of the metallo-hydrolase superfamily. In eubacterium as well as mitochondria and chloroplasts, the protein synthesis starts from formylmethioninetRNA, resulting in the polypeptides with $\mathrm{N}$-terminals formylated ${ }^{1}$. During the elongation of the polypeptide chain, the formyl group is removed hydrolytically by $\mathrm{PDF}^{2-4}$, which may be a universally conserved feature of the prokaryotic protein synthesis and an essential component for bacterial survival ${ }^{5}$. Actually, due to the continuous increase of the tolerance to conventional antibiotics, the synthesis of new and effective drugs is imperative. PDF is an attractive target for the design of new antibiotics because at present, the deformylation does not occur in the cytoplasm of eucaryotic cells ${ }^{6}$.

Previous researches showed that the activity of Leptospira interrogans PDF ( $L i \mathrm{PDF}$ ), the bacterial zinc PDF with a high activity, is strongly dependent on both $\mathrm{pH}$ values and metal ions. The catalytic activity of the $L i \mathrm{PDF}$ decreases for $\mathrm{pH}<7.0$ and is nearly constant in the $\mathrm{pH}$ range from 7.5 to $10.5^{7}$. Moreover, in many macromolecules both structures and functions are also affected by $\mathrm{pH}$, a parameter associated with the concentration of hydrogen ions. The majority of proteins exhibit an optimal $\mathrm{pH}$, but why and how the biological activity of proteins depends by $\mathrm{pH}$ remains an open problem, in particular at the atomic scale. Different $L i \mathrm{PDF}$ structures at different $\mathrm{pH}$ values are available in the protein crystallography databank (PDB code: $1 \mathrm{Y} 6 \mathrm{H} \mathrm{pH3.0,1VEV} \mathrm{pH6.5,} \mathrm{1VEY}$ $\mathrm{pH} 7.0,1 \mathrm{SV} 2 \mathrm{pH} 7.5,1 \mathrm{VEZ} \mathrm{pH8.0)^{8 }}$. Nevertheless, the biochemical mechanism of the $\mathrm{pH}$-dependent enzymatic behavior has never been clearly demonstrated mainly because, within the error bars of the crystallography structures, folds of the active sites in $L i \mathrm{PDFs}$ are substantially identical.

On the basis of the presence of a classical HEXXH zinc-binding motif, PDF was originally thought to be a zinc enzyme. On the contrary, PDFs utilize different metal ions in different organisms ${ }^{9}$. Different from Escherichia coli $\mathrm{PDF}(E c \mathrm{PDF})$ in which the $\mathrm{Co}^{2+}$-bound form (Co-EcPDF) is active, while the $\mathrm{Zn}^{2+}$-bound form ( $\left.\mathrm{Zn}-E c \mathrm{PDF}\right)$ is inactive ${ }^{8}$, the $\mathrm{Co}^{2+}$-bound $L i \mathrm{PDF}(\mathrm{Co}-L i \mathrm{PDF})$ and the $\mathrm{Ni}^{2+}$-bound $L i \mathrm{PDF}(\mathrm{Ni}-L i \mathrm{PDF})$ are less active than $\mathrm{Zn}-$ $L i \mathrm{PDF}^{10}$. Although the catalytic mechanism is unknown, Jain et al. pointed out that at the end of the catalytic reaction, the metal ion in the active site might be penta- or tetra-coordinated ${ }^{11}$. However, the LiPDF with the 
native $\mathrm{Zn}$ ion shares the same coordination sphere in the crystal structure of the inactive $\mathrm{Zn}$-PDFs, not in agreement with the enzymatic activity scenario proposed by Jain $e t$ al..

In the active centre of most PDFs, metal ions are coordinated with the side chains of one cysteine and two histidines plus a water molecule. The latter plays a significant role in the deformylate reaction, performing the nucleophilic attack on the carbonyl carbon of the formyl group which leads to the transition state of the reaction ${ }^{12}$. This was turned out to be the rate-limiting $\operatorname{step}^{13}$. The reaction scheme is schematically described in Fig. 1. A similar situation occurs in other metallohydrolases ${ }^{14-16}$ pointing out that to explore and understand the catalytic activity and the reaction mechanism is mandatory to study water molecules in the reaction center.

$\mathrm{X}$-ray absorption spectroscopy on biological systems (BioXAS) is a short-range probe sensitive to local structure variations, extremely useful to reconstruct in details and understand the environment of selected atoms in biological systems ${ }^{17-19}$. BioXAS allows investigating samples in a wide variety of forms, such as proteins as oriented single crystals, disoriented micro-crystals, frozen or room-temperature solutions or proteins inside membranes. XAS includes the extended X-ray absorption fine structure (EXAFS) and the X-ray absorption near-edge structure (XANES). The EXAFS analysis is reliable and provides the average distance between metal and its ligands while XANES is able to obtain three-dimensional structural information, although a quantitative analysis is still challenging in complex systems. Here, we present and discuss XANES spectra at the $\mathrm{Zn}$ and Co K-edge of $L i \mathrm{PDF}$ and EcPDF vs. pH. Combining ab initio multiple scattering calculations, we applied the MXAN package to analyze XANES data and extract subtle local structural changes around metal sites in LiPDF at different $\mathrm{pH}$ values and in the $E c \mathrm{PDF}$ at optimum $\mathrm{pH}$, describing with particular emphasis the location and geometry of bonded water molecules.

\section{Results and Discussion}

Correlation between structure, function and $\mathrm{pH}$. $\mathrm{Zn} \mathrm{K}$-edge experimental XANES spectra of $L i \mathrm{PDF}$ at different $\mathrm{pH}$ values are compared in Fig. 2. In the right panel we compare the differences of XANES spectra of each solution with the spectrum at $\mathrm{pH} 8.5$, the mid value of the range in which the protein has the highest activity, taken as the reference.

We may recognize that differences in the $\mathrm{pH}$ range from 7.0 to 11.0 are extremely small, while larger differences occur for $\mathrm{pH}$ values $<$ 7.0, particularly in the edge region $(-10 \sim 20 \mathrm{eV})$. As it is well known, XAS features in this region are due to multiple scattering contributions from the nearest neighbours and reflect both the geometric and the electronic structure of the probed system. Because for a $\mathrm{Zn}^{2+}$ ion the $3 d$ orbital are completely filled, the main peak at the $\mathrm{Zn}$ $K$-edge reflects the local geometrical configuration in an accurate way. In particular, the splitting of the main peak at the $\mathrm{Zn} \mathrm{K}$-edge points out the presence of two different chemical environments involving the metal ion.

In order to obtain accurate structural information around the metal site, based on the crystallographic structure of the LiPDF ( $\mathrm{PDB}$ code $1 \mathrm{Y} 6 \mathrm{H}(\mathrm{B})$ ), we performed a quantitative analysis of the XANES spectrum using the MXAN code ${ }^{20}$. Experimental XANES spectra (black) of the $L i$ PDF vs. $\mathrm{pH}$ and best-fit calculations (red dots) are compared in Fig. 3. A good agreement occurs among experimental data and best-fit theoretical curves for the whole energy range. All features in the near-edge region are successfully reproduced. Using alternative starting structures the calculations returned the same results. The refined main distances of the best fits of the structures vs. $\mathrm{pH}$ are summarized in Table 1 . The statistical errors of independent parameters with the certain $R_{\mathrm{sq}}$ values were evaluated by the MIGRAD subroutine of MXAN code ${ }^{21}$. The error values of

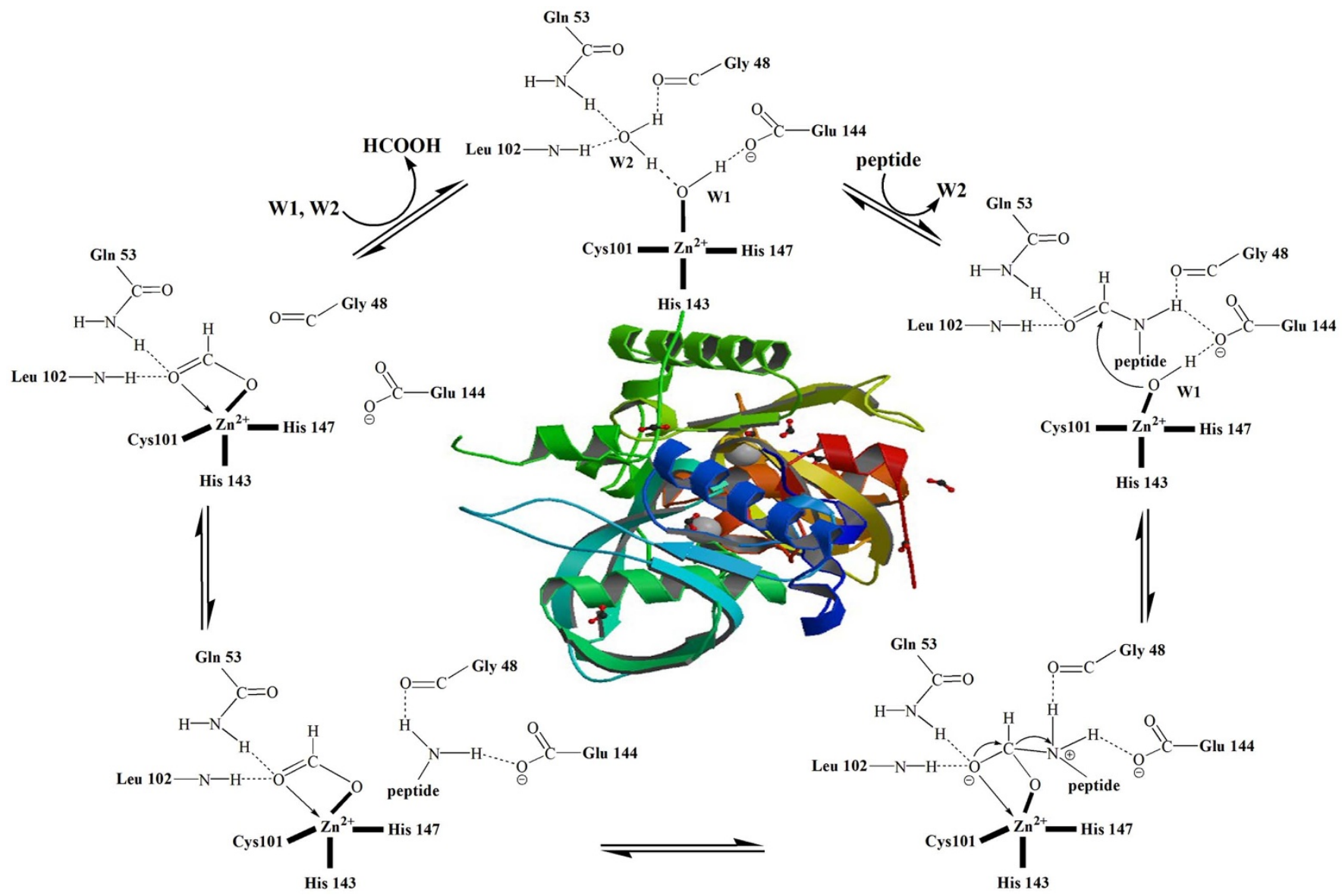

Figure $1 \mid$ The action scheme for $\mathrm{PDF}^{12}$ and inside the crystal structure of PDF (PDB code: 1Y6H). 


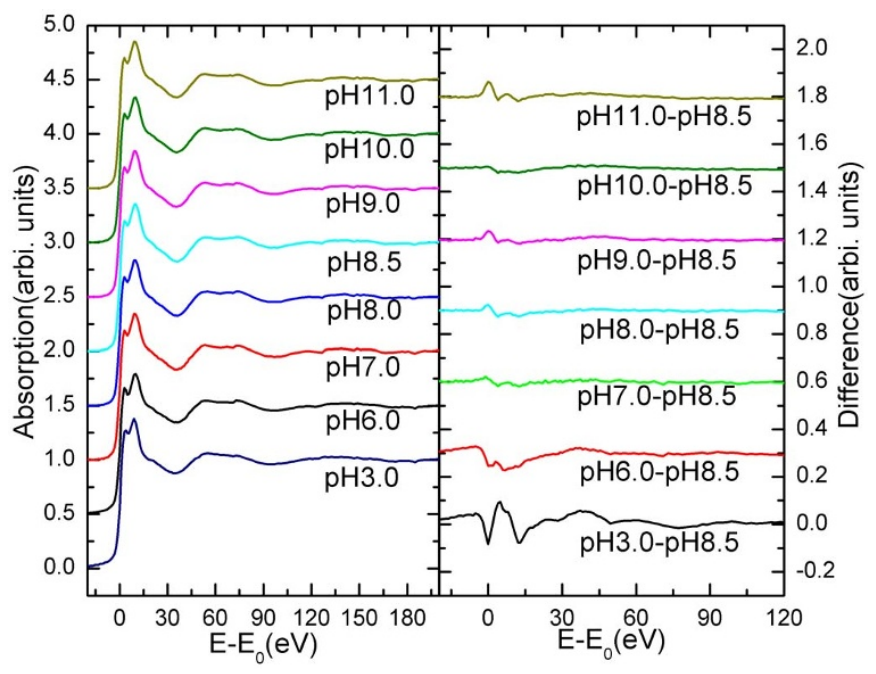

Figure 2 | Left: Zn K-edge XANES spectra of the LiPDF vs. pH; Right: comparison among differences between $\mathrm{Zn}$ K-edge XANES spectra and the reference spectrum collected at $\mathrm{pH}$ 8.5. Differences are negligible except at the edge and in particular for $\mathrm{pH}$ values: 3 and 6 .

Wat1-Wat2 and Wat1-Glu44 were estimated from the independent parameters of Zinc-Wat1, Zinc-Wat2 and Zinc-Glu44.

From Table 1 it is evident that the most significant differences among local structures at different $\mathrm{pH}$ is the bond length between the zinc ion and two water molecules, a condition that points out the key role for water. In the native state of $L i \mathrm{PDF}$ at $\mathrm{pH} 8.5$, the Watl coordinating with the $\mathrm{Zn}$ ion $(2.50 \AA)$ is connected to the Wat $2(2.97 \AA)$ and the side chain oxygen of the Glu144 (2.50 $\AA$ ) by hydrogen bonds. The side chain of the conserved Leu102 donates protons to the Wat 2 (2.59 $\AA$ ) molecule, which forms other hydrogen bonds with the amide in the main chain of $\operatorname{Gln} 53(3.43 \AA)$ and the oxygen in the main chain of Gly 48

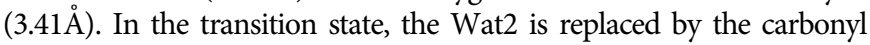
oxygen of the formyl group ${ }^{12}$. The comparison of the local structure of the $L i \mathrm{PDF}$ at $\mathrm{pH} 8.5$ (best fit - coloured sticks) and the crystallographic model structure (thick yellow sticks) is shown in Fig. 4.

Outlined in Fig. 5, we also show the correlation between the zincWat1-distance (bond distance between the $\mathrm{Zn}$ ion and the Wat1 molecule) and $\mathrm{pH}$. We hypothesize that the enzymatic activity mechanism induced by $\mathrm{pH}$ is associated to the changes in the $\mathrm{Zn}$-Watl bond distance that affects the ionization degree of the Watl molecule. In the catalytic process the nucleophilic addition of metalbound water to the carbonyl of the $\mathrm{N}$-terminal formyl of the peptide substrate is indeed a crucial step. The metal acts as an electronwithdrawing group helping the deprotonation of the Wat1, as well as a Lewis acid activating the carbonyl bond with the substrate ${ }^{12}$. In an acid environment ( $\mathrm{pH} 3-\mathrm{pH}$ 6), with the increase of the $\mathrm{Zn}-\mathrm{Wat} 1-$ distance, the proton of the Watl is more localized and the charge

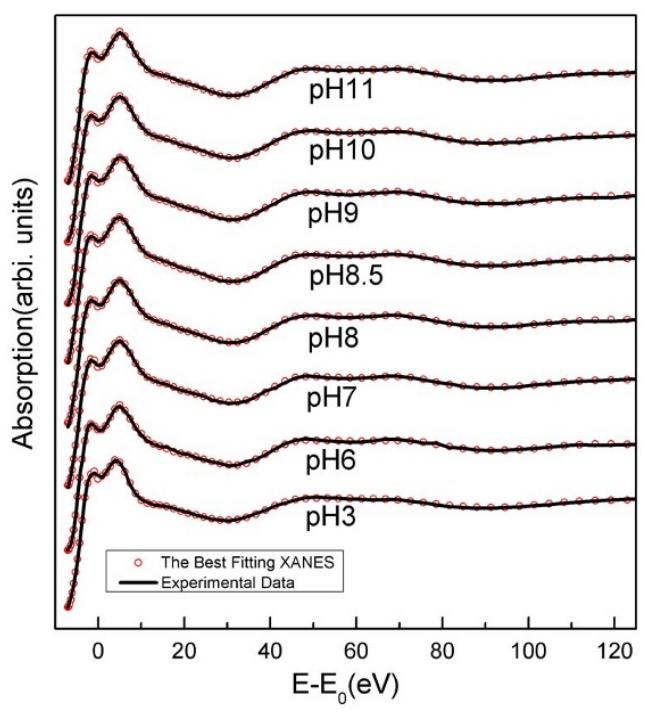

Figure 3 Comparison among experimental Zn K-edge XANES spectra vs. pH (black) and simulations (red dot).

transfer towards the amide at the N-terminus of the peptide, even with the help of Glu144, is more difficult. This mechanism may explain why the enzymatic activity of $L i P D F$ is much lower in an acid environment.

Correlation between structure, function and metal-dependence. We also investigated the metal-dependent enzymatic activity of PDFs. The Zn K-edge XANES spectra of Zn-EcPDF (red) and Zn-LiPDF (blue), the Co K-edge XANES spectra of Co-EcPDF (black) and Co-LiPDF (green) in a buffer solution at $\mathrm{pH} 8.0$ are compared in Fig. 6. The peak $\mathrm{A}$ is due to multiple-scattering events among the nearest neighbours and reflects the geometry as well as the electronic structure of the systems. In view of the different electronic configurations of $\mathrm{Zn}^{2+}$ and $\mathrm{Co}^{2+}$, a simple comparison of the main peak $\mathrm{A}$ has no meaning. Moreover, because of the unoccupied $3 d$ orbital of the $\mathrm{Co}^{2+}$, a pre-edge peak (peak $\mathrm{P}$ ) is observed in the XANES spectra of both Co- $\mathrm{L}$ PDF and Co-EcPDF. This feature is dipole-forbidden in a centro-symmetric configuration that is an allowed quadrupole electronic transitions ( $1 s$ to $3 d$ transition). For the absorbed $3 d$ metal with local spherical symmetry (such as with hexagon coordinators), this transition is forbidden. When the spherical symmetry breaks, such as ligands distorting and coordinator number decreasing, the XANES will present a weak pre-edge peak. As reported in previous work ${ }^{22,23}$, the weak pre-edge peak suggests a distorting penta-coordination structure with the low electronegative coordinating atoms.

We performed a quantitative analysis of XANES spectra with the MXAN package. According to the above, Fig. 7 and Table 2 show that the most significant difference in the local structure around metal

Table 1 | Refined critical distances of the best fit at different $\mathrm{pH}$

\begin{tabular}{|c|c|c|c|c|c|c|c|c|c|}
\hline Atom pairs & $\begin{array}{c}\text { Model } \\
\text { structure(Å) }\end{array}$ & $\begin{array}{c}\mathrm{pH} 3.0 \\
\text { distance }(\AA)\end{array}$ & $\begin{array}{c}\mathrm{pH6.0} \\
\text { distance( }(\AA)\end{array}$ & $\begin{array}{c}\mathrm{pH} 7.0 \\
\text { distance( }(\dot{A})\end{array}$ & $\begin{array}{c}\text { pH8.0 } \\
\text { distance( }(\dot{A})\end{array}$ & $\begin{array}{c}\mathrm{pH} 8.5 \\
\text { distance( }(\AA)\end{array}$ & $\begin{array}{c}\mathrm{pH} 9.0 \\
\text { distance( }(\stackrel{\mathrm{A}}{)})\end{array}$ & $\begin{array}{c}\mathrm{pH} 10.0 \\
\text { distance( }(\AA)\end{array}$ & $\begin{array}{c}\mathrm{pH} 11.0 \\
\text { distance( }(\AA)\end{array}$ \\
\hline $\begin{array}{l}R_{\text {sq }} \text { (square } \\
\text { residue) }\end{array}$ & - & 0.453 & 0.245 & 0.599 & 0.466 & 0.362 & 0.450 & 0.377 & 0.507 \\
\hline Zinc His 147 & 2.15 & $2.06 \pm 0.02$ & $2.01 \pm 0.02$ & $2.02 \pm 0.02$ & $2.02 \pm 002$ & $2.02 \pm 0.02$ & $2.03 \pm 0.02$ & $2.03 \pm 0.03$ & $1.99 \pm 0.02$ \\
\hline Zinc His 143 & 2.07 & $2.06 \pm 0.02$ & $2.10 \pm 0.02$ & $2.04 \pm 0.02$ & $2.05 \pm 0.02$ & $2.05 \pm 0.02$ & $2.04 \pm 0.02$ & $2.04 \pm 0.02$ & $2.07 \pm 0.02$ \\
\hline Zinc Cys 101 & 2.40 & $2.25 \pm 0.03$ & $2.25 \pm 0.03$ & $2.25 \pm 0.03$ & $2.23 \pm 0.03$ & $2.24 \pm 0.03$ & $2.23 \pm 0.03$ & $2.23 \pm 0.03$ & $2.26 \pm 0.04$ \\
\hline Zinc Wat 1 & 2.31 & $2.58 \pm 0.02$ & $2.57 \pm 0.02$ & $2.52 \pm 0.02$ & $2.50 \pm 0.02$ & $2.50 \pm 0.02$ & $2.50 \pm 0.02$ & $2.52 \pm 0.02$ & $2.53 \pm 0.02$ \\
\hline Zinc Wat2 & 3.90 & $4.05 \pm 0.05$ & $3.91 \pm 0.04$ & $3.97 \pm 0.04$ & $4.00 \pm 0.04$ & $4.00 \pm 0.04$ & $4.00 \pm 0.04$ & $4.05 \pm 0.04$ & $3.92 \pm 0.04$ \\
\hline Wat1 Wat2 & 3.20 & $2.59 \pm 0.06$ & $3.00 \pm 0.05$ & $2.99 \pm 0.05$ & $3.05 \pm 0.05$ & $2.97 \pm 0.05$ & $2.95 \pm 0.05$ & $2.99 \pm 0.06$ & $2.87 \pm 0.05$ \\
\hline Watl & 2.67 & $2.57 \pm 0.07$ & $2.51 \pm 0.06$ & $2.54 \pm 0.07$ & $2.52 \pm 0.06$ & $2.50 \pm 0.06$ & $2.48 \pm 0.06$ & $2.52 \pm 0.06$ & $2.56 \pm 0.07$ \\
\hline Glu144 & & & & & & & & & \\
\hline
\end{tabular}




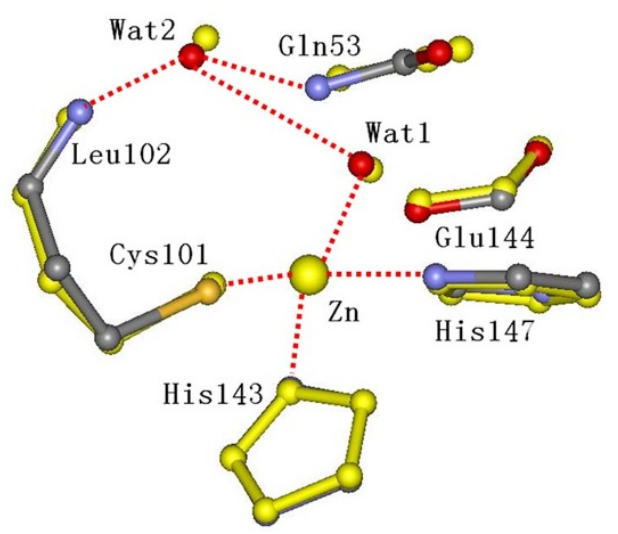

Figure 4 | Comparison between the local structure of the best fit at $\mathrm{pH} 8.5$ (colored sticks) and the crystallographic model structure (thick yellow sticks).

ions in $\mathrm{Co} / \mathrm{Zn}-L i \mathrm{PDF}$ and $\mathrm{Co} / \mathrm{Zn}-E c \mathrm{PDF}$ is the length of the metalWat1 bond. The Co-Watl distance in Co- LiPDF is $1.91 \AA$, much shorter than in $\mathrm{Zn}-\mathrm{LiPDF}(2.50 \AA)$. However, the Co-Wat1 distance of Co-EcPDF is $2.15 \AA$, significantly longer than in Zn-EcPDF (2.05 $\AA$ ). Actually, the strong binding with water inhibits the transition of the metal ion from a penta- to tetra-coordination in the catalytic process and increases the distance between water and Glu144/133 (Wat1-Glu). This mechanism weakens the polarization of Glu144/ 133 to Wat 1 and prevents the transfer of a proton from Wat to the substrate, the key step in the catalytic process of PDFs ${ }^{12}$. Thus, the strong binding of the metal-Watl reduces the polarizability of Glu144/133 towards Wat 1 and significantly decreases the probability of the transfer of a proton from the Watl to the amide at the Nterminus, which actually takes into account the reduction of the enzymatic activity of Co- LiPDF and $\mathrm{Zn}-E c \mathrm{PDF}$ with respect to $\mathrm{Zn}$ $L i \mathrm{PDF}$ and Co-EcPDF.

Summarizing, XAS is a powerful tool to resolve the local structure around the metal atom in biological samples at low concentration, although the EXAFS method may hardly detect subtle structural changes. XANES data show that PDF may exhibit a high enzymatic

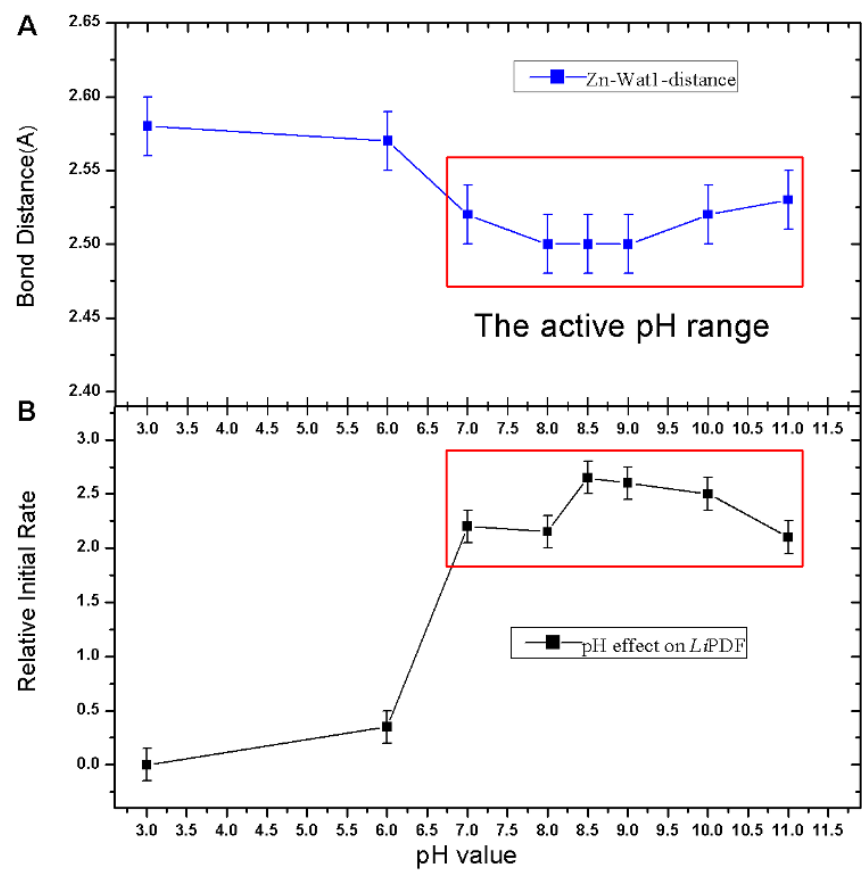

Figure 5 | Correlation between the $\mathrm{Zn}$-Wat1 distance vs. $\mathrm{pH}(\mathrm{A})$ and the activity of the LiPDF vs. pH (B).

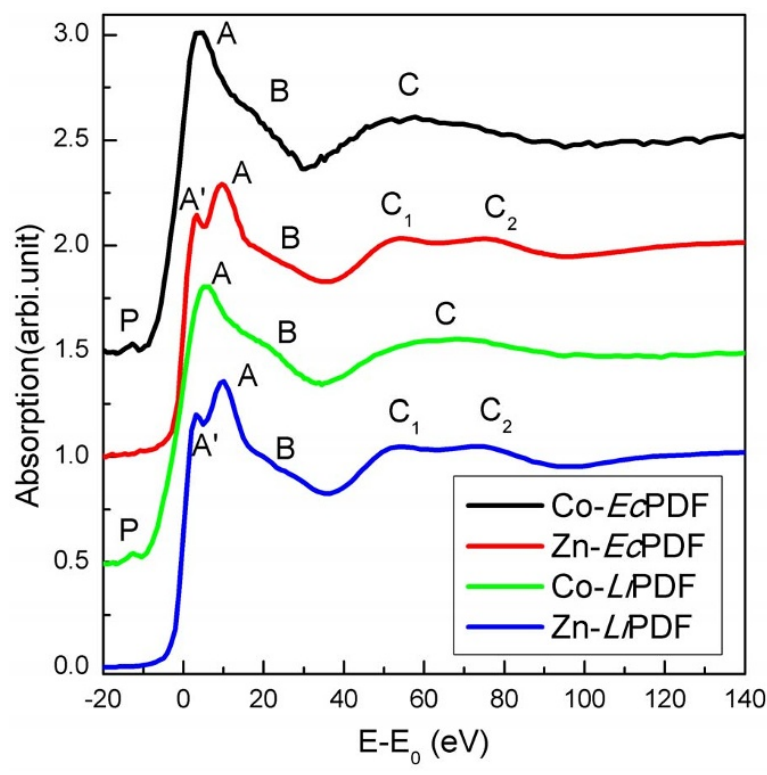

Figure $6 \mid \mathrm{Zn}$ K-edge XANES spectra of Zn-EcPDF (red) and Zn-LiPDF (blue) and Co K-edge XANES spectra of Co-EcPDF (black) and CoLiPDF (green) in a buffer solution at $\mathrm{pH}$ 8.0.

activity when the nearest metal coordinating water molecule is localised at a distance from the metal ion in the range $2.15-2.55 \AA$, while a low enzymatic activity can be associated to metal-Wat 1 distances outside this range, i.e., longer than $2.55 \AA$ or shorter than $2.15 \AA$.

\section{Conclusion}

This work is an accurate investigation of $\mathrm{pH}$ - and metal-dependent enzymatic activity of the peptide deformylase. Local structures around the central metal ions were characterized using XANES spectroscopy combined with $a b$ initio calculations. All features in the near-edge region of the spectra were successfully reproduced and detailed structural information around the metal ion was obtained. Data clearly point out that the enzymatic activity depends on $\mathrm{pH}$ values and metal ions via the metal-Watl distance. Actually, the proton of the Wat1 is localized and the charge transfer towards the amide is blocked with the extremely long metal-Watl distance in an acid environment $(\mathrm{pH}<$ 6). However, a too short metal-Watl distance induces a strong binding with water, which inhibits the transition of the metal ion from a penta- to tetra-coordination in the catalytic process.

From a careful comparison among structures of other PDFs, we may also claim that PDF exhibits a high enzymatic activity when the Wat1 is located within a limited range of distance, i.e., between 2.15 and $2.55 \AA$ from the metal ion. Data provide accurate structural information and point out a real novel scenario regarding the mechanism responsible of the observed $\mathrm{pH}$ dependent enzymatic activity. The new unique structural information appears useful to trigger further applications of these proteins, focused in particular on the development of new drugs and more effective antibiotics.

\section{Methods}

E.coli BL21(DE3) cells carrying plasmid pET22b- $L i$ PDF were expressed as reported in Ref. 7. Cells were harvested by centrifugation and resuspended in $25 \mathrm{ml}$ of buffer A (50 mM Tris- $\mathrm{HCl}$ pH7.5, $10 \mathrm{mM} \mathrm{NaCl}$ ). After sonication cell debris were removed by centrifugation. The supernatant $(25 \mathrm{ml})$ was applied onto a HiTrapTM $5 \mathrm{ml} \mathrm{Q} \mathrm{HP}$ (Amersham Pharmacia Biotech) column equilibrated with buffer B (50 mM Tris$\mathrm{HCl} \mathrm{pH} 8.5,10 \mathrm{mM} \mathrm{NaCl}$ ). The column was eluted with $250 \mathrm{ml}$ of buffer B plus a linear gradient of $10 \mathrm{mM}-1 \mathrm{M} \mathrm{NaCl}$ (AKTA purifier 900, Amersham Pharmacia Biotech). The fractions with $L i P D F$ activity were concentrated and applied to a SuperdexTM 75 column (16/60,Amersham Pharmacia Biotech) pre-equilibrated with buffer $\mathrm{C}(50 \mathrm{mM}$ Tris- $\mathrm{HCl} \mathrm{pH} 8.5,50 \mathrm{mM} \mathrm{NaCl})$. The fractions containing LiPDF activity were collected. The sample homogeneity was monitored by SDSPAGE and the protein concentration was determined by Bradford assay with BSA as the standard. $E c P D F$ was purified using the same procedures. 

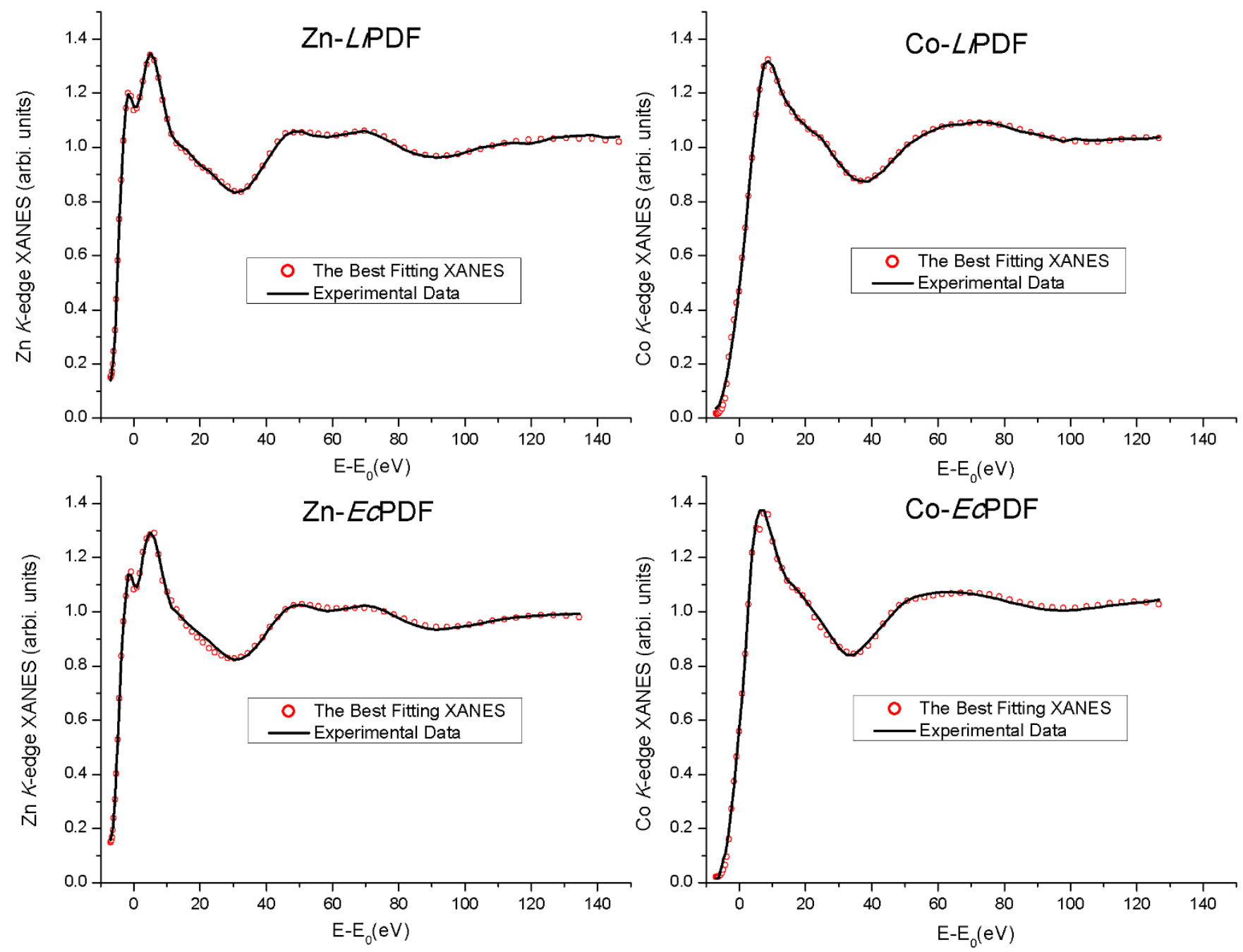

Figure 7 | Comparison among best fits of XANES spectra (red dots) and experimental spectra (black lines) in buffer solutions at pH 8.0 of (A) ZnLiPDF; (B) Co-LiPDF; (C) Zn-EcPDF and (D) Co-EcPDF.

To acquire samples of $L i \mathrm{PDF}$ at different $\mathrm{pH}$ values, we used the purification protocol as described above, by direct dialysis exchanges respectively with $50 \mathrm{mM}$ $\mathrm{NaHCO}_{3} / \mathrm{Na}_{2} \mathrm{CO}_{3}$ (pH 9.0, pH 10, pH 11), 50 mM Tris- $\mathrm{HCl}$ ( $\mathrm{pH} 7.5, \mathrm{pH} 8.0$ ), $50 \mathrm{mM} \mathrm{NaH}_{2} \mathrm{PO}_{4} / \mathrm{Na}_{2} \mathrm{HPO}_{4}$ (pH 6.0, pH 7.0) and $50 \mathrm{mM} \mathrm{Gly-HCl}$ (pH 3.0). All PDF concentrations were about $60 \mathrm{mg} / \mathrm{ml}$ and the $\mathrm{Zn}^{2+}$ and $\mathrm{Co}^{2+}$ concentrations were around 200 ppm.

$\mathrm{X}$-ray absorption spectroscopy measurements at the $\mathrm{Zn}$ and $\mathrm{Co} K$-edges were carried out at the beam line 4W1B of the Beijing Synchrotron Radiation Facility (BSRF) and the beam line 14W1 of the Shanghai Synchrotron Radiation Facility (SSRF) in the fluorescence yield mode at room temperature. The typical energy of the storage ring was $2.5 \mathrm{GeV}$ in BSRF and $3.5 \mathrm{GeV}$ in SSRF and experiments were performed with a decreasing electron current from $250 \mathrm{~mA}$ to $160 \mathrm{~mA}$. The incident beam intensity was monitored using an ionization chamber flowed by a $25 \%$ argon-doped nitrogen mixture while the fluorescence signal was collected by means of a Lytle detector flowed by argon gas. As previously described, after the experiments, we recycled the samples and measured their enzymatic activity ${ }^{7}$. Data showed that the activity of proteins is preserved.

In order to extract structural/geometrical information around the metal site, we performed a quantitative analysis of XANES spectra using the MXAN package. This

Table 2 | Refined critical distances and error bars of the best fits of Zn-LiPDF, Co-LiPDF, Zn-EcPDF and Co-EcPDF

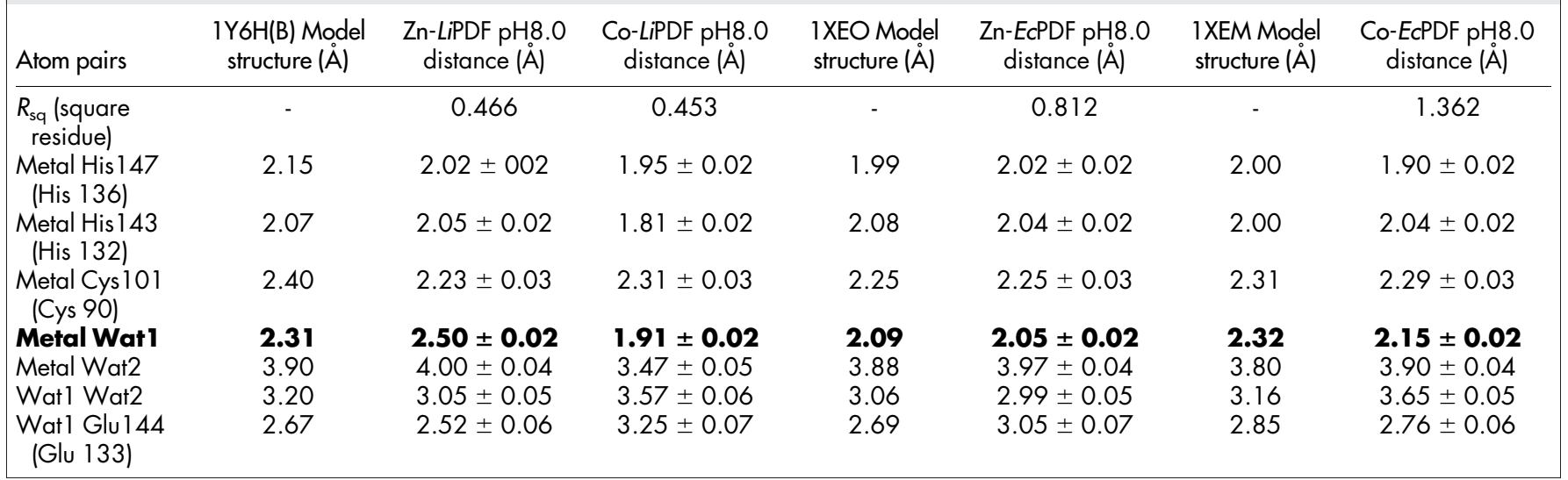


software is capable of performing a quantitative analysis of a XANES spectrum from the absorption edge up to $\sim 200 \mathrm{eV}$ via comparison between experimental data and theoretical calculations obtained by changing relevant geometrical parameters around the photon absorber site ${ }^{24,25}$. The X-ray absorption cross sections were calculated using the full multiple scattering approach in the framework of the muffin-tin (MT) approximation for the shape of the potential ${ }^{26,27}$. In this case, exchange and correlation parts of the potential were determined on the basis of the local density approximation of the self energy. Inelastic processes were taken into account by a convolution with a broadening Lorentzian function having an energy-dependent width of the form $\Gamma(E)=\Gamma_{\mathrm{c}}+\Gamma_{\mathrm{mfp}}(E)$ in which the constant part $\Gamma_{\mathrm{c}}$ takes care of both the core-hole lifetime $(1.8 \mathrm{eV})^{28}$ and the experimental resolution $(1.5 \mathrm{eV})$, while the energy-dependent term represents intrinsic and extrinsic inelastic processes ${ }^{25,29,30}$. The method takes into account Multiple-Scattering events in a rigorous way through the evaluation of the scattering path operator ${ }^{26,31}$. Its reliability has been successfully tested over the years in a wide number of different applications ${ }^{24,29}$. In this case spectra typically converge when the cluster includes 39 atoms within a radius of $5.5 \AA$ A. Therefore the local structure of the PDF active center may be properly represented by an atomic cluster containing fifty atoms within $6 \AA$ from the metal ion.

1. Meinnel, T., Mechulam, Y. \& Blanquet, S. Methionine as translation start signal - a review of the enzymes of the pathway in Escherichia-coli. Biochimie. 75, 1061-1075 (1993).

2. Adams, J. M. On release of formyl group from nascent protein. J. Mol. Biol. 33, 571-589 (1968).

3. Ball, L. A. \& Kaesberg, P. Cleavage of N-terminal formylmethionine residue from a bacteriophage coat protein in-vitro. J. Mol. Biol. 79, 531-537 (1973).

4. Livingston, D. M. \& Leder, P. Deformylation and protein biosynthesis. Biochemistry 8, 435-443 (1969).

5. Mazel, D., Pocher, S. \& Marliere, P. Genetic-characterization of polypeptide deformylase, a distinctive enzyme of eubacterial translation. EMBO J. 13, 914-923 (1994).

6. Rajagopalan, P. T. R., Yu, X. C. \& Pei, D. H. Peptide deformylase: a new type of mononuclear iron protein. J. Am. Chem. Soc. 119, 12418-12419 (1997).

7. Li, Y. K., Chen, Z. F. \& Gong, W. M. Enzymatic properties of a new peptide deformylase from pathogenic bacterium Leptospira interrogans. Biochem. Bioph. Res. Co. 295, 884-889 (2002).

8. Zhou, Z. C., Song, X. M. \& Gong, W. M. Novel conformational states of peptide deformylase from pathogenic bacterium Leptospira interrogans - Implications for population shift. J. Biol. Chem. 280, 42391-42396 (2005).

9. Nguyen, K. T., Wu, J. C., Boylan, J. A., Gherardini, F. C. \& Pei, D. Zinc is the metal cofactor of Borrelia burgdorferi peptide deformylase. Arch. Biochem. Biophys. 468, 217-225 (2007).

10. Li, Y. K., Ren, S. X. \& Gong, W. M. Cloning, high-level expression, purification and crystallization of peptide deformylase from Leptospira interrogans. Acta. Cryst. D 58, 846-848 (2002).

11. Jain, R. K., Hao, B., Liu, R. P. \& Chan, M. K. Structures of E.coli peptide deformylase bound to formate: Insight into the preference for $\mathrm{Fe}^{2+}$ over $\mathrm{Zn}^{2+}$ as the active site metal. J. Am. Chem. Soc. 127, 4558-4559 (2005).

12. Becker, A. et al. Iron center, substrate recognition and mechanism of peptide deformylase. Nat. Struct. Mol. Bio. 5, 1053-1058 (1998).

13. Chen, S. L., Li, Z. S. \& Fang, W. H. Theoretical investigation of astacin proteolysis. J. Inorg. Biochem. 111, 70-79 (2012).

14. Yoshimoto, T. et al. Crystal structures of creatininase reveal the substrate binding site and provide an insight into the catalytic mechanism. J. Mol. Biol. 337, 399-416 (2004).

15. Gennadios, H. A., Whittington, D. A., Li, X. C., Fierke, C. A. \& Christianson, D. W. Mechanistic inferences from the binding of ligands to LpxC, a metal-dependent deacetylase. Biochemistry. 45, 7940-7948 (2006).

16. Yamashita, K. et al. Substitution of Glu122 by glutamine revealed the function of the second water molecule as a proton donor in the binuclear metal enzyme creatininase. J. Mol. Biol. 396, 1081-1096 (2010).

17. Nordlander, E. et al. Heterometal cuboidal clusters $\mathrm{MFe}_{4} \mathrm{~S}_{6}\left(\mathrm{PEt}_{3}\right)_{4} \mathrm{Cl}(\mathrm{M}=\mathrm{V}, \mathrm{Mo})$ - synthesis, structural-analysis by crystallography and EXAFS, and relevance to the core structure of the iron molybdenum cofactor of nitrogenase. J. Am. Chem. Soc. 115, 5549-5558 (1993).

18. Conradson, S. D. et al. Selenol binds to iron in nitrogenase iron-molybdenum cofactor: an extended x-ray absorption fine structure study. Proc. Natl. Acad. Sci. 91, 1290-1293 (1994).

19. Stone, K. L., Behan, R. K. \& Green, M. T. X-ray absorption spectroscopy of chloroperoxidase compound I: Insight into the reactive intermediate of P450 chemistry. Proc. Natl. Acad. Sci. 102, 16563-16565 (2005).
20. Zhou, Z. C., Song, X. M., Li, Y. K. \& Gong, W. M. Unique Structural Characteristics of Peptide Deformylase from Pathogenic Bacterium Leptospira interrogans. J. Mol. Biol. 339, 207-215 (2004),

21. Chillemi, G. et al. Evidence for Sevenfold Coordination in the First Solvation Shell of $\mathrm{Hg}$ (II) Aqua Ion. J. Am. Chem. Soc. 129, 5430-5436 (2007)

22. Grunes, L. A. Study of the $K$ edges of $3 d$ transition metals in pure and oxide form by x-ray-absorption spectroscopy. Phys. Rev. B 27, 2111-2131 (1983)

23. Wu, Z. Y., Ouvrard, G., Gressier, P. \& Natoli, C. R. Ti and O K edges for titanium oxides by multiple scattering calculations: Comparison to XAS and EELS spectra. Phys. Rev. B 55, 10382-10391 (1997)

24. Della Longa, S., Arcovito, A., Girasole, M., Hazemann, J. L. \& Benfatto, M. Quantitative analysis of $\mathrm{x}$-ray absorption near edge structure data by a full multiple scattering procedure: the Fe-CO geometry in photolyzed carbonmonoxy-myoglobin single crystal. Phys. Rev. Lett. 87, 155501 (2001).

25. Cardelli, A. et al. A crystal-chemical investigation of $\mathrm{Cr}$ substitution in muscovite by XANES spectroscopy. Phys. Chem. Miner. 30, 54-58 (2003).

26. Natoli, C. R. \& Benfatto, M. A unifying scheme of interpretation of $\mathrm{x}$-ray absorption-spectra based on the multiple-scattering theory. J. Phys. (Paris) Colloq. 47, 11-23 (1986)

27. Wu, Z. Y. et al. Sulfur $K$-edge $\mathrm{x}$-ray-absorption study of the charge transfer upon lithium intercalation into titanium disulfide. Phys. Rev. Lett. 77, 2101-2104 (1996).

28. Fuggle, J. C. \& Inglesfield, J. E. Unoccupied electronic states - fundamentals for XANES, EELS, IPS and BIS - introduction. Top Appl. Phys. 69, 1-23 (1992).

29. Benfatto, M., Della Longa, S. \& Natoli, C. R. The MXAN procedure: a new method for analysing the XANES spectra of metalloproteins to obtain structural quantitative information. J. Synchrotron Radiat. 10, 51-57 (2003).

30. Roscioni, O. M., D’Angelo, P., Chillemi, G., Della Longa, S. \& Benfatto, M. Quantitative analysis of XANES spectra of disordered systems based on molecular dynamics. J. Synchrotron Radiat. 12, 75-79 (2005).

31. Wu, Z. Y. et al. Symmetry dependence of X-ray absorption near-edge structure at the metal $K$ edge of $3 d$ transition metal compounds. Appl. Phys. Lett. 79, $1918-1920$ (2001).

\section{Acknowledgments}

We are very grateful to A. Marcelli for useful advices on the early draft of this manuscript. We sincerely acknowledge the staff of the XAS beamline of BSRF and SSRF for the technology supports in the XAS experiments. This work was partly supported by the Knowledge Innovation Program of the Chinese Academy of Sciences (KJCX2-YW-N42), the States Key Project for Fundamental Research (2012CB825801), the Key Important Project of the National Natural Science Foundation of China (10734070), the National Natural Science Foundation of China (NSFC 11275227 \& 10905067) and the Science Fund for Creative Research Groups (11321503).

\section{Author contributions}

Z.W. and W.G. designed the experiments. P.C., Y.W., X.G. and M.Y. performed experiments and data analysis. P.C., Y.W., W.C., F.Y., H.Z. and Z.W. performed the calculations. Y.D. and Y.X. supplied some biological experiment apparatus. W.G. and Z.W. led the whole work and the analysis. P.C., Y.W., W.C. and Z.W. wrote the text. All authors reviewed the manuscript

\section{Additional information}

Competing financial interests: The authors declare no competing financial interests.

How to cite this article: Cui, P. et al. How water molecules affect the catalytic activity of hydrolases - A XANES study of the local structures of peptide deformylase. Sci. Rep. 4, 7453; DOI:10.1038/srep07453 (2014)

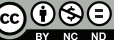

This work is licensed under a Creative Commons Attribution-NonCommercialNoDerivs 4.0 International License. The images or other third party material in this article are included in the article's Creative Commons license, unless indicated otherwise in the credit line; if the material is not included under the Creative Commons license, users will need to obtain permission from the license holder in order to reproduce the material. To view a copy of this license, visit http:// creativecommons.org/licenses/by-nc-nd/4.0/ 